\title{
Investigating source confusion in PMN J1603-4904
}

\author{
F. Krauß ${ }^{1}$, M. Kreter ${ }^{2}$, C. Müller ${ }^{3}$, A. Markowitz ${ }^{4,5}$, M. Böck ${ }^{6}$, T. Burnett ${ }^{7}$, T. Dauser ${ }^{6}$, M. Kadler ${ }^{2}$, \\ A. Kreikenbohm ${ }^{2}$, R. Ojha ${ }^{8}$, and J. Wilms ${ }^{6}$
}

\begin{abstract}
${ }^{1}$ GRAPPA \& Anton Pannekoek Institute for Astronomy, University of Amsterdam, Science Park 904, 1098 XH Amsterdam, The Netherlands e-mail: Felicia.Krauss@uva.nl

2 Institut für Theoretische Physik und Astrophysik, Universität Würzburg, Emil-Fischer-Str. 31, 97074 Würzburg, Germany

${ }^{3}$ Department of Astrophysics/IMAPP, Radboud University Nijmegen, Heyendaalseweg 135, 6525 AJ Nijmegen, The Netherlands

${ }^{4}$ Nicolaus Copernicus Astronomical Center, Polish Academy of Sciences, Bartycka 18, 00-716 Warszawa, Poland

${ }^{5}$ Center for Astrophysics \& Space Science, University of California, San Diego, La Jolla, CA 92093-0424, USA

${ }^{6}$ Dr. Remeis Sternwarte \& ECAP, Universität Erlangen-Nürnberg, Sternwartstrasse 7, 96049 Bamberg, Germany

7 Department of Physics, University of Washington, Seattle, WA 98195-1560, USA

${ }^{8}$ NASA, Goddard Space Flight Center, Astrophysics Science Division, Code 661, Greenbelt, MD 20771, USA
\end{abstract}

Received 22 November 2017 / Accepted 3 January 2018

\begin{abstract}
PMN J1603-4904 is a likely member of the rare class of $\gamma$-ray emitting young radio galaxies. Only one other source, PKS 1718-649, has been confirmed so far. These objects, which may transition into larger radio galaxies, are a stepping stone to understanding AGN evolution. It is not completely clear how these young galaxies, seen edge-on, can produce high-energy $\gamma$ rays. PMN J1603-4904 has been detected by TANAMI Very Long Baseline Interferometry (VLBI) observations and has been followed-up with multiwavelength observations. A Fermi Gamma-ray Space Telescope Large Area Telescope (Fermi-LAT) $\gamma$-ray source has been associated with this young galaxy in the LAT catalogs. We have obtained Chandra observations of the source to consider the possibility of source confusion due to the relatively large positional uncertainty of Fermi-LAT. The goal was to investigate the possibility of other X-ray bright sources in the vicinity of PMN J1603-4904 that could be counterparts to the $\gamma$-ray emission. With Chandra/ACIS, we find no other sources in the uncertainty ellipse of Fermi-LAT data, which includes an improved localization analysis of eight years of data. We further study the X-ray fluxes and spectra. We conclude that PMN J1603-4904 is indeed the second confirmed $\gamma$-ray bright young radio galaxy.
\end{abstract}

Key words. galaxies: active - galaxies: jets - Galaxy: abundances - galaxies: individual: PMN J1603-4904

\section{Introduction}

Active galactic nuclei (AGN) are the most luminous persistent objects in the Universe. A subset of AGN exhibits relativistic outflows, called jets. Many questions of jet physics remain unsolved, including the details of jet launching, confinement, and acceleration processes. In this context, peculiar AGN in transitory stages become relevant for addressing the key questions of AGN science. Among these objects are young radio galaxies, which exhibit shorter jets (up to a few kpc), and are also known as compact symmetric objects (CSO) owing to their compactness at radio wavelengths (Phillips \& Mutel 1982; Wilkinson et al. 1994; Readhead et al. 1996; O’Dea 1998). Young radio galaxies are typically seen at large inclination angles to the jet(s) and have negligible Doppler boosting. While $\gamma$-ray emission from young AGN was predicted (Kino et al. 2007, 2009; Stawarz et al. 2008; Kino \& Asano 2011), a detection by the Fermi Gamma-ray Space Telescope Large Area Telescope (hereafter Fermi-LAT) remained elusive for many years (D'Ammando et al. 2016). Using the improved Pass 8 reconstruction (Atwood et al. 2013), Migliori et al. (2016) detected PKS 1718-649, which was the first CSO at $\gamma$-ray energies. Three other candidate sources have been proposed: 4C+55.17 (McConville et al. 2011), PKS 1413+135 (Gugliucci et al. 2005), and PMN J1603-4904 (Müller et al. 2014). The first two have not been confirmed to be young radio galaxies. It remains unclear which attributes make some young AGN $\gamma$-ray loud. A direct link between narrow-line Seyfert 1 galaxies (NLS1) and young AGNs (compact symmetric sources) has been suggested by Caccianiga et al. (2014), but seems unlikely (Orienti et al. 2015).

PMN J1603-4904 is a radio source (Wright et al. 1994). It was recently confirmed to be a CSO from $\mathrm{MHz}$ data, which makes it a young or frustrated AGN (Müller et al. 2016). This source was detected by Fermi-LAT and classified as a low synchrotron peaked (LSP) BL Lac object (2FGL J1603.8-4904, 3FGL J1603.9-4903; Nolan et al. 2012; Acero et al. 2015; Ackermann et al. 2015). The first association with the $\gamma$-ray source was proposed by Kovalev (2009). It is also listed in the LAT catalogs of sources detected above $10 \mathrm{GeV}$ and $50 \mathrm{GeV}$ (1FHL J1603.7-4903, 2FHL J1603.94903, and 3FHL J1603.8-4903; Ackermann et al. 2013, 2016; The Fermi-LAT Collaboration2017). PMN J1603-4904 is reported as a variable source in the 3FGL catalog. PMN J1603-4904 was first proposed to be a $\gamma$-ray bright CSO by Müller et al. (2014), who discussed its unusual VLBI structure and spectral energy distribution (SED). We followed up on this paper with X-ray observations with Suzaku and XMM-Newton, which led to the first high S/N X-ray spectrum that also exhibited an emission line at $5.44 \mathrm{keV}$, which we interpreted as a redshifted neutral Fe $\mathrm{K} \alpha$ line (at $z=0.18$; Müller et al. 2015). Optical data by Shaw et al. (2013), which resulted in the LSP BL Lac classification, were not sensitive enough to detect any lines. 
Further optical spectroscopy showed our proposed redshift to be incorrect. X-shooter data resulted in a redshift measurement of $z=0.2321 \pm 0.0004$ (Goldoni et al. 2016). The emission line is due to $\mathrm{He}$-like $\mathrm{Fe}$, emitted at a rest-frame energy of $6.7 \mathrm{keV}$. This emission feature is not typically seen in AGN, where edgeon sources exhibit neutral or slightly ionized $\mathrm{Fe} \mathrm{K} \alpha$ emission, which is expected to originate in the accretion disks. This feature seems to be common for CSO sources (Siemiginowska et al. 2016). Highly ionized Fe emission is also observed in the LINER galaxy M 81 (Page et al. 2004), although the latter still exhibits neutral $\mathrm{Fe} \mathrm{K} \alpha$ emission while PMN J1603-4904 does not. It is unclear whether this suggests a complete lack of an accretion disk, a truncated accretion disk (which could likely achieve temperatures sufficiently high to ionize Fe) or a lack of neutral Fe.

Further radio studies of PMNJ1603-4904 find a lowfrequency turnover in the $\mathrm{MHz}-\mathrm{GHz}$ spectrum, which indicates a source extent of $1.4 \mathrm{kpc}$ and confirmed its young radio source classification (Müller et al. 2016). The source is located close to the Galactic plane $\left(l=332^{\circ} .15, b=2.57\right)$, which hinders optical/UV and soft X-ray observations due to extinction and photoelectric absorption. The low Galactic latitude also complicates $\gamma$-ray data analysis because of the large number of nearby sources, the Galactic diffuse emission, and the three nearby extended sources, which all have to be taken into account. The radio source is consistent with the Fermi-LAT 95\% 2FGL positional uncertainty, but it is unclear if there are other possible counterparts within the uncertainty ellipse in either radio or X-ray wavelengths (Müller et al. 2014). Optical data are unable to solve this problem because of the large number of nearby stars and the strong extinction. In this paper we examine recent Chandra/ACIS data, the highest angular resolution data available at high energies, to confirm or rule out source confusion for PMN J1603-4904. In Sect. 2 we discuss the observations and analysis methods. In Sect. 3 we discuss the results of the observations. The final section reports our conclusions.

\section{Observations and methods}

\subsection{Chandra}

We took one Chandra observation of PMN J1603-4904 with the Advanced CCD Imaging Spectrometer (ACIS; observation ID $17106,10.08 \mathrm{ksec}$ ) on 12 May 2016. The data are not affected by pile-up, as PMNJ1603-4904 is a relatively weak X-ray emitter (Müller et al. 2015). The data were extracted using the standard tools from the CIAO 4.8. The extraction radius for PMN J1603-4904 is 3.'3, while the background was extracted with annuli centered on the source position with radii of 4". 4 and $40^{\prime \prime}$. The source to the east of PMN J1603-4904 (no. 1, see Fig. 1) was extracted with an extraction radius of 4".9, while the background annulus radii were $8 . .3$ and $25^{\prime \prime} .9$. The source to the east is seen in archival XMM data as well. The source to the west is not detected in XMM, either because of its low flux or because it is variable. The spectral analysis was performed with the Interactive Spectral Interpretation System (ISIS, version 1.6.2-40; Houck \& Denicola 2000). The Chandra data were modeled with an absorbed power law, which fits both PMN J1603-4904 and the eastern source (no. 1). Because of low $\mathrm{S} / \mathrm{N}$ in the individual bins, we used Cash statistics (Cash 1979) for spectral analysis. For the absorbing column we used the abundances of Wilms et al. (2000) and the cross sections of Verner et al. (1996) with the newest version of the tbnew model ${ }^{1}$. In order to determine

\footnotetext{
1 Available online at: http://pulsar.sternwarte. uni-erlangen.de/wilms/research/tbabs/
}

the coordinates of the X-ray sources we ran the CIAO tools mkps fmap (at $2 \mathrm{keV}$ with $50 \%$ enclosed counts) and wavdetect with the default scales. For the dereddening of the optical/UV/IR data, the best-fit absorbing column $N_{\mathrm{H}}$ was converted to $A_{\mathrm{V}}$ from X-ray dust scattering halo measurements of Predehl \& Schmitt (1995); Nowak et al. (2012) have updated the revised abundance of the interstellar medium (see Krauß et al. 2016, for a detailed explanation of the treatment of multiwavelength data).

\subsection{Fermi-LAT $\gamma$-ray data analysis}

For the analysis of the Fermi-LAT $\gamma$-ray data, we used the Fermi Science Tools (v11r0p0) with the reprocessed Pass 8 data and the P8R2_SOURCE_V6 instrument response functions. The localization of the source is tricky with gtfindsrc because of its position near the Galactic plane. The localization we performed is similar to that in the 3FGL catalog (Acero et al. 2015) and only data above $3.2 \mathrm{GeV}$ were used as the data were binned four per decade. The log-likelihood surface is assumed to be parabolic. A selection of eight points is sampled in a circle around the estimated position, which is used to estimate the five parameters for the ellipse. The center is then moved to the estimated maximum, a new circle chosen at the $2 \sigma$ radius, and the procedure is iterated until convergence. The deviation of the fit from the measured values defines a goodness-of-fit quantity. The curvature of the surface is used to determine the covariance matrix, which in turn determines the positional uncertainty ellipse. We quote the values corresponding to a $95 \%$ containment. The positions and uncertainties are then used to determine the need for systematic adjustment by comparing with a set of AGNs, which have very accurate radio positions. To account for the systematics, we multiply the $95 \%$ uncertainties by a factor of 1.05 , and add $0.433^{\prime}$ in quadrature. For the spectral analysis we used an unbinned likelihood analysis in a region of interest of $5^{\circ}$ around PMN J1603-4904 in the 1-300 GeV energy range. Sources within a $15^{\circ}$ radius of PMN J1603-4904 were included in the likelihood fitting and their parameters were fixed. A free spectral index is used, together with a detection threshold of test statistic $T S=25$ (Wilks 1938).

\section{Results}

We find three X-ray sources in the Chandra/ACIS image in the direct vicinity of the 2MASS coordinates of PMN J1603-4904 (see Fig. 1; Skrutskie et al. 2006). The coordinates are given in Table 1. The X-ray source in the center (no. 2) matches the radio coordinates. We can exclude the western source (no. 3) as a counterpart. It is at an angular distance of 2!.9 to PMN J1603-4904, well outside the uncertainty ellipses of our analysis and those of the Fermi-LAT catalogs. The eastern source (no. 1) is also outside the LAT uncertainty ellipses, but closer, because this source is at an angular distance of 1.7 and the 3FGL uncertainty region seems to be closer to the eastern source than any of the LAT results (see Fig. 1, right panel). Based on this image, the $\gamma$-ray source is indeed the counterpart to the young radio source, although the radio source lies just outside the 3FGL 95\% uncertainty ellipse. In the eight-year Fermi-LAT analysis, the source is detected at $T S=2373$, with a photon index $\Gamma=1.98 \pm 0.03$ and a flux $F_{1-300 \mathrm{GeV}}=(5.57 \pm 0.22) \times 10^{-9} \mathrm{ph} \mathrm{s}^{-1} \mathrm{~cm}^{-2}$. This flux is slightly lower than the fluxes reported in the LAT catalogs (Fig. 2).

We further analyze the Chandra spectra of PMN J1603-4904 and of the source to the east of PMN J1603-4904. X-ray observations by Suzaku and XMM-Newton were taken from Müller et al. (2015). The indices 

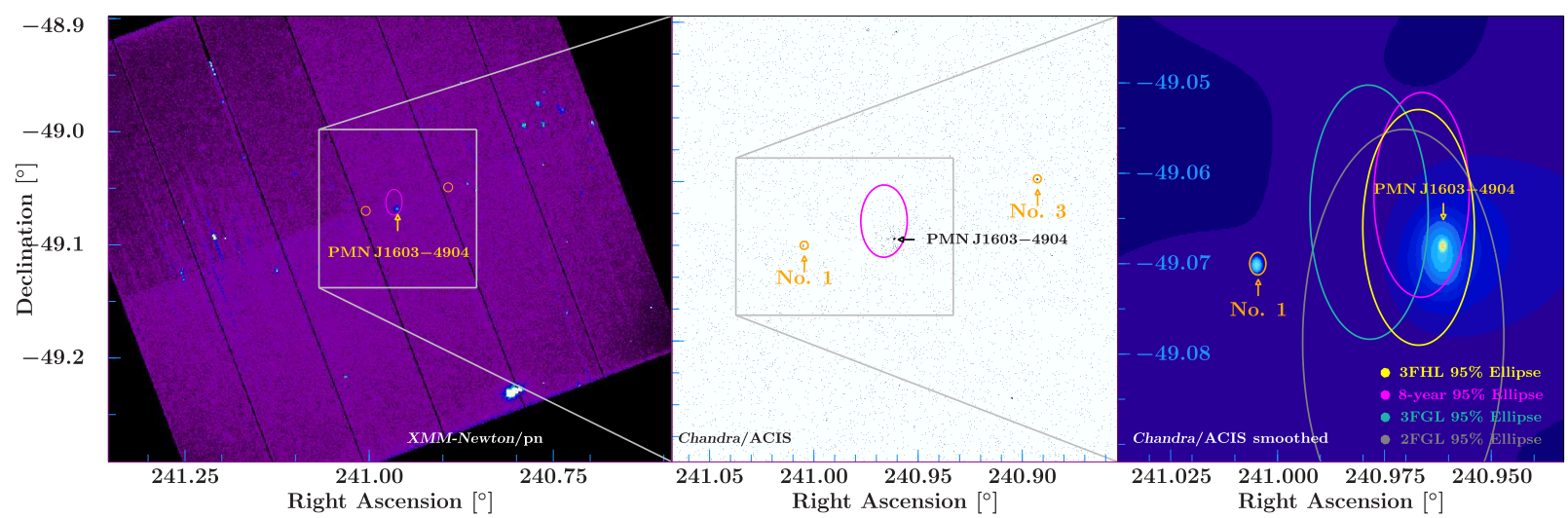

Fig. 1. XMM-Newton and Chandra/ACIS images of PMN J1603-4904. The positions of the sources are indicated with arrows. Two unknown, weak X-ray sources are shown with orange circles. The 95\% uncertainty on the Fermi-LAT positions from the 2FGL, 3FGL, and the 3FHL catalogs are represented with gray, green, and yellow, respectively. Left: XMM-Newton/pn observation (ObsID 0724700101); middle: Chandra/ACIS observation (ObsID 17106); right: same Chandra/ACIS observation, the image was smoothed with a Gaussian kernel of S/N range of 3-5.

Table 1. Chandra positions of the three X-ray sources in the direct vicinity of PMN J1603-4904 $\left(\alpha_{\mathrm{J} 2000.0}=16^{\mathrm{h}} 03^{\mathrm{m}} 50^{\mathrm{s}} .69, \delta_{\mathrm{J} 2000.0}=\right.$ $\left.-49^{\circ} 04^{\prime} 05^{\prime \prime} \cdot 49\right)$

\begin{tabular}{lcccc}
\hline \hline No. & $\alpha_{\mathrm{J} 2000.0}$ & $\delta_{\mathrm{J} 2000.0}$ & $u\left(\alpha_{\mathrm{J} 2000.0}\right)$ & $u\left(\delta_{\mathrm{J} 2000.0}\right)$ \\
\hline 1 & $16^{\mathrm{h}} 04^{\mathrm{m}} 01^{\mathrm{s}} .111$ & $-49^{\circ} 04^{\prime} 12^{\prime \prime} .00$ & $0.139^{\prime \prime}$ & $0.182^{\prime \prime}$ \\
2 & $16^{\mathrm{h}} 03^{\mathrm{m}} 50^{\mathrm{s}} .687$ & $-49^{\circ} 04^{\prime} 04^{\prime \prime} 44$ & $0.046^{\prime \prime}$ & $0.035^{\prime \prime}$ \\
3 & $16^{\mathrm{h}} 03^{\mathrm{m}} 34.280$ & $-49^{\circ} 02^{\prime} 57^{\prime \prime} .16$ & $0.238^{\prime \prime}$ & $0.130^{\prime \prime}$ \\
\hline
\end{tabular}

Notes. Positions and uncertainties were determined using wavdetect. The position of the central source (no. 2) is consistent with the radio position of PMN J1603-4904.

Table 2. Best fit values from Suzaku and XMM-Newton data taken from Müller et al. (2015) in comparison with best fit values for Chandra/ACIS data for PMN J1603-4904 and two fits to the eastern source.

\begin{tabular}{lcccc}
\hline \hline Parameter & $\begin{array}{c}\text { Suzaku \& } \\
\text { XMM }\end{array}$ & Chandra & $\begin{array}{c}\text { Chandra } \\
\text { East }\end{array}$ & $\begin{array}{c}\text { Chandra } \\
\text { East }\end{array}$ \\
\hline$N_{\mathrm{H}}$ & $2.05_{-0.12}^{+0.14}$ & 2.05 & 2.05 & 0.632 \\
$\Gamma$ & $2.07_{-0.12}^{+0.04}$ & $2.23_{-0.28}^{+0.29}$ & $5.3_{-2.1}^{+1.5}$ & $3.0_{-1.2}^{+1.4}$ \\
$F_{2-10}$ & $4.39 \pm 0.17$ & $2.8_{-0.6}^{+0.7}$ & $0.08_{-0.04}^{+0.05}$ & $0.14_{-0.1}^{+0.28}$ \\
\hline$\chi^{2} /$ d.o.f. & $183.0 / 162$ & $28.8 / 37$ & $2.9 / 3$ & $2.3 / 3$ \\
\hline
\end{tabular}

Notes. Values without uncertainties have been frozen to the given value. Uncertainties are given at the $90 \%$ confidence level. The absorbing column is given in units of $10^{22} \mathrm{~cm}^{-2}$ and the unabsorbed $2-10 \mathrm{keV}$ flux is given in units of $10^{-13} \mathrm{erg} \mathrm{s}^{-1} \mathrm{~cm}^{-2}$.

are consistent with the Chandra best fit of PMN J1603-4904, while the flux is slightly lower (see Table 2). Because of the low $\mathrm{S} / \mathrm{N}$, particularly at energies above $5 \mathrm{keV}$, the Fe emission line is not detected with Chandra. We model the Chandra/ACIS data with an absorbed power law and with an absorbed collisionally ionized emission model (APEC) and compare the results to those of the Suzaku/XMM-Newton data from 2013 (see Table 3).

It is worth noting that the eastern source has a very soft index of $\Gamma=5.3_{-2.1}^{+1.5}$, assuming the same absorbing column as for PMN J1603-4904. With a $21 \mathrm{~cm}$ derived Galactic equivalent hydrogen column of $6.32 \times 10^{21} \mathrm{~cm}^{-2}$ (Kalberla et al. 2005) the index is much flatter and more realistic. This suggests that the source has little or no intrinsic absorption. We add the Chandra data to the high-energy SED, which includes the combined Suzaku and XMM data and the LAT spectrum from the 3FGL catalog (see Fig. 3). We have included both the spectrum from PMN J1603-4904 and from the eastern source (in black and

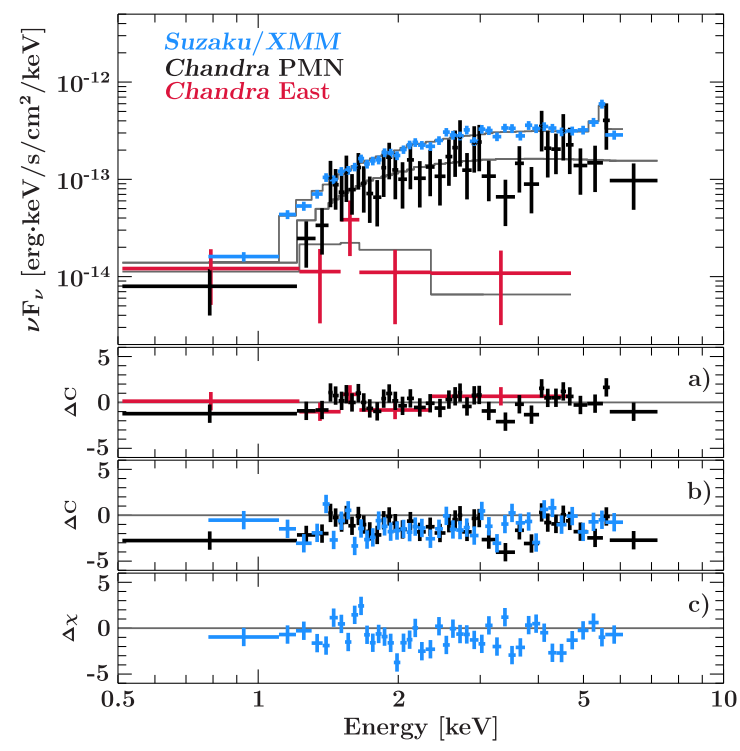

Fig. 2. X-ray spectra: Combined Suzaku/XMM-Newton spectrum of PMNJ1603-4904 (blue), Chandra/ACIS spectrum of PMN J1603-4904 (black), and Chandra/ACIS spectrum of the source east of PMNJ1603-4904 (pink). A best-fit absorbed power law is shown for the two Chandra spectra. The fit models shown are the best fit power-law models for the Chandra spectrum and the APEC model for the combined Suzaku/XMM data. Residuals are given for the a) best fit power-law spectrum, $b$ ) the combined APEC fit, and $c$ ) the Suzaku/XMM APEC fit.

pink, respectively). While it is already challenging to explain the strong $\gamma$-ray emission and the flat $\gamma$-ray index in combination with the flat X-ray index of PMN J1603-4904 (in both Suzaku $+\mathrm{XMM}$ and Chandra), it is nearly impossible to explain the soft index of the eastern source in combination with the LAT data, except by invoking different populations of particles. The high-energy SED seems to confirm that the eastern source is an extremely unlikely counterpart. Modeling the broadband SED of PMN J1603-4904 with a physical one-zone model will remain challenging with such a high Compton dominance and flat indices.

Although the optical/UV is likely nonthermal (Goldoni et al. 2016), a further possibility is thermal emission from, for example, an APEC component, which would explain the He-like Fe line; this explanation is consistent with results by Siemiginowska et al. (2016). A combined fit to the Chandra/ACIS and combined Suzaku and XMM data results in 


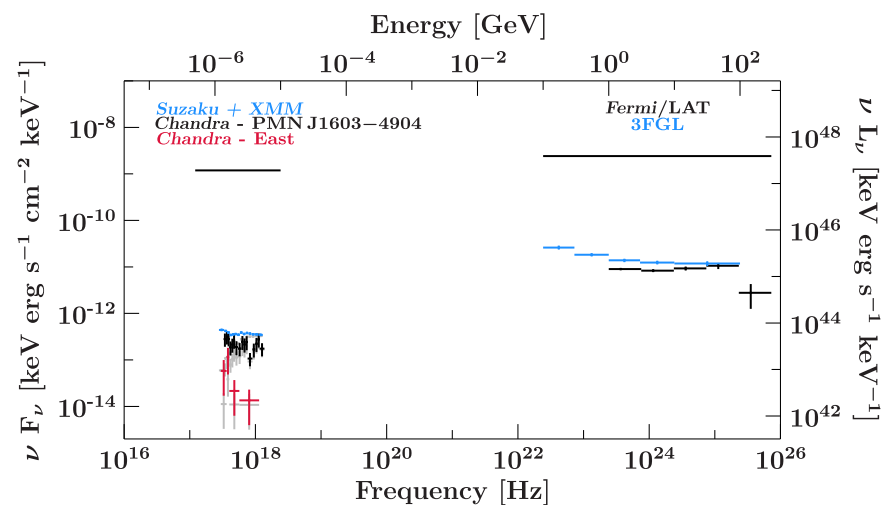

Fig. 3. High-energy SED showing the archival combined Suzaku and XMM data and the Chandra/ACIS data of both PMN J1603-4904 and the eastern source in X-rays. The Fermi-LAT spectrum of PMN J1603-4904 is shown. The absorbed X-ray spectra are shown in gray.

Table 3. APEC best fit values to combined fitting of Chandra/ACIS and Suzaku/XMM data, and only Suzaku/XMM data.

\begin{tabular}{lllll}
\hline \hline Fit & $\begin{array}{l}N_{\mathrm{H}} \\
{\left[10^{22} \mathrm{~cm}^{-2}\right]}\end{array}$ & $\begin{array}{l}\mathrm{kT} \\
{[\mathrm{keV}]}\end{array}$ & Abundance & $\chi^{2} /$ d.o.f. \\
\hline comb. & $1.62 \pm 0.12$ & $5.9_{-0.7}^{+0.9}$ & $0.46_{-0.14}^{+0.16}$ & $137.298 / 78$ \\
XMM & $1.61 \pm 0.13$ & $6.1_{-0.8}^{+1.1}$ & $0.46_{-0.16}^{+0.17}$ & $31.816 / 39$ \\
\hline
\end{tabular}

Notes. The fit to the combined data sets was carried out using Cash statistic and not $\chi^{2}$ statistics, therefore $\chi^{2}$ is the Cash statistic value in that case.

a best fit absorbing column of $(1.62 \pm 0.12) \times 10^{22} \mathrm{~cm}^{-2}$, which can explain the absence of observed He-like S/Si. It is interesting to note that this value is lower than the value necessary for a purely phenomenological power-law fit with an added Gaussian line, which is $2.05_{-0.12}^{+0.14} \times 10^{22} \mathrm{~cm}^{-2}$. This lower value is not compatible with the Galactic absorption of $6.32 \times 10^{21} \mathrm{~cm}^{-2}$ and suggests intrinsic absorption, possibly from a dusty torus, which is in agreement with the interpretation of the blackbody feature in the infrared with a hot torus (Müller et al. 2014).

Photoionization is an alternative way to produce the He-like $\mathrm{Fe}$ emission line, but the low $\mathrm{S} / \mathrm{N}$ of the spectra does not allow us to differentiate between the models.

\section{Conclusions}

We have presented Chandra/ACIS and Fermi-LAT data of PMN J1603-4904. We show that we can rule out source confusion between the Fermi-LAT source and sources at lower energies by using the high angular resolution of Chandra and an improved eight-year localization of the $\gamma$-ray source 3FGL J1603.9-4903. The positional uncertainty is consistent with the radio coordinates and the X-ray counterpart. An alternative X-ray counterpart at $\sim 1^{\prime}$ distance to PMN J1603-4904 can be ruled out as a counterpart to the $\gamma$-ray source based on the localization and spectral shape. Its spectral index is further inconsistent with LAT data, as seen from the high-energy SED. Its absorbing column is consistent with Galactic absorption, although the low $\mathrm{S} / \mathrm{N}$ makes a good fit of the absorbing column impossible. Radio observations from the ATPMN catalog agree with the conclusions of this paper, where no other source is seen in the vicinity of PMN J1603-4904 (McConnell et al. 2012).

Using Chandra/ACIS positions, fluxes, and spectra, in combination with Fermi-LAT data, we confirm the X-ray counterpart, rule out contributions from nearby X-ray sources, and confirm a high Compton dominance in the broadband SED of PMNJ1603-4904. We therefore conclude that PMN J1603-4904 is likely one of only two known $\gamma$-ray emitting young radio galaxies and that its emission mechanisms and strong Compton dominance warrant further research.

Acknowledgements. We thank the anonymous referee for helpful comments. We thank F. D'Ammando as the internal LAT referee and A. Dominguez, E. Cavazutti, and D. Thompson for helpful comments which have greatly improved the paper. We thank M. Hanke for the help with the projections of the TS maps. F. K. acknowledges funding from the European Union's Horizon 2020 research and innovation program under grant agreement No. 653477. C.M. acknowledges funding from the ERC Synergy Grant "BlackHoleCam: Imaging the Event Horizon of Black Holes" (Grant 545 610058). We thank J. E. Davis for the development of the slxfig module that has been used to prepare the figures in this work. This research has made use of a collection of ISIS scripts provided by the Dr. Karl Remeis-Observatory, Bamberg, Germany at http://www.sternwarte. uni-erlangen.de/isis/. The Fermi-LAT Collaboration acknowledges support for LAT development, operation and data analysis from NASA and DOE (United States), CEA/Irfu and IN2P3/CNRS (France), ASI and INFN (Italy), MEXT, KEK, and JAXA (Japan), and the K.A. Wallenberg Foundation, the Swedish Research Council and the National Space Board (Sweden). Science analysis support in the operations phase from INAF (Italy) and CNES (France) is also gratefully acknowledged. This work performed in part under DOE Contract DE-AC02-76SF00515.

\section{References}

Acero, F., Ackermann, M., Ajello, M., et al. 2015, ApJS, 218, 23 Ackermann, M., Ajello, M., Allafort, A., et al. 2013, ApJS, 209, 34 Ackermann, M., Ajello, M., Atwood, W. B., et al. 2015, ApJ, 810, 14 Ackermann, M., Ajello, M., Atwood, W. B., et al. 2016, ApJS, 222, 5 Atwood, W., Albert, A., Baldini, L., et al. 2013, in 2012 Fermi Symposium eConf C121028

Caccianiga, A., Antón, S., Ballo, L., et al. 2014, MNRAS, 441, 172

Cash, W. 1979, ApJ, 228, 939

D'Ammando, F., Orienti, M., \& Giroletti, M. 2016, Fermi Large Area Telescope Collaboration 2016, Astron. Nachr., 337, 59

Goldoni, P., Pita, S., Boisson, C., et al. 2016, A\&A, 586, L2

Gugliucci, N. E., Taylor, G. B., Peck, A. B., \& Giroletti, M. 2005, ApJ, 622, 136 Houck, J. C., \& Denicola, L. A. 2000, in Astronomical Data Analysis Software and Systems IX, eds. N. Manset, C. Veillet, \& D. Crabtree, ASP Conf. Ser. 216, 591

Kalberla, P. M. W., Burton, W. B., Hartmann, D., et al. 2005, A\&A, 440, 775

Kino, M., \& Asano, K. 2011, MNRAS, 412, L20

Kino, M., Kawakatu, N., Ito, H. 2007, MNRAS, 376, 1630

Kino, M., Ito, H., Kawakatu, N., \& Nagai, H., 2009, MNRAS, 395, L43

Kovalev, Y. Y. 2009, ApJL, 707, L56

Krauß, F., Wilms, J., Kadler, M., et al. 2016, A\&A, 591, A130

McConnell, D., Sadler, E.M., Murphy, T., Ekers, R. D., 2012, MNRAS, 422, 1527

McConville, W., Ostorero, L., Moderski, R., et al. 2011, ApJ, 738, 148

Migliori, G., Siemiginowska, A., Sobolewska, M., et al. 2016, ApJ, 821, L31

Müller, C., Kadler, M., Ojha, R., et al. 2014, A\&A, 562, A4

Müller, C., Krauß, F., Dauser, T., et al. 2015, A\&A, 574, A117

Müller, C., Burd, P. R., Schulz, R., et al. 2016, A\&A, 593, L19

Nolan, P. L., Abdo A. A., Ackermann, M., et al. 2012, ApJS, 199, 31

Nowak, M. A., Neilsen, J., Markoff, S. B., et al. 2012, ApJ, 759, 95

O’Dea, C. P. 1998, PASP, 110, 493

Orienti, M., D’Ammando, F., Larsson, J., et al. 2015, MNRAS, 453, 4037

Page, M. J., Soria, R., Zane, S., et al. 2004, A\&A, 422, 77

Phillips, R. B., \& Mutel, R. L., 1982, A\&A, 106, 21

Predehl, P., Schmitt, J. H. M. M., 1995, A\&A, 293, 889

Readhead, A. C. S., Taylor, G. B., Pearson, T. J., \& Wilkinson, P. N., 1996, ApJ, 460,634

Shaw, M. S., Romani, R. W., Cotter, G., et al. 2013, ApJ, 764, 135

Siemiginowska, A., Sobolewska, M., Migliori, G., et al. 2016, ApJ, 823, 57

Skrutskie, M. F., Cutri, R. M., Stiening, R., et al. 2006, AJ, 131, 1163

Stawarz, Ł., Ostorero, L., Begelman, M. C., et al. 2008, ApJ, 680, 911

The Fermi-LAT Collaboration 2017, ApJS, 232, 18

Verner, D. A., Ferland, G. J., Korista, K. T., \& Yakovlev, D. G. 1996, ApJ, 465, 487

Wilkinson, P. N., Polatidis, A. G., Readhead, A. C. S., et al. 1994, ApJ, 432, L87

Wilks, S. S. 1938, Ann. Math. Statist., 9, 60

Wilms, J., Allen, A., McCray, R. 2000, ApJ, 542, 914

Wright, A. E., Griffith, M. R., Burke, B. F., \& Ekers, R. D. 1994, ApJS, 91,111 